\title{
Enfoque Multinivel para el Diagnóstico de la Actividad Física en Tres Regiones de Colombia
}

Adriana Prieto-Rodríguez ${ }^{1}$ y Carlos A. Agudelo-Calderon ${ }^{2}$

${ }^{1}$ Fisioterapeuta. M. Sc. Salud Pública. Departamento del Movimiento Corporal Humano, Facultad de Medicina. Universidad Nacional.

E-mail: aprietor@unal.edu.co

${ }^{2}$ Medico. M. Sc. Salud Pública, M. Sc. Ciencias. Instituto de Salud Pública, Facultad de Medicina. Universidad Nacional de Colombia. E-mail: caagudeloc@unal.edu.co

Recibido 10 Agosto 2005/Enviado para Modificación 14 Diciembre 2005/Aceptado 31 Enero 2006

RESUMEN

Objetivo Se estudiaron características individuales y contextuales relacionadas con la actividad física, para describir e interpretar ésta práctica en tres regiones colombianas.

Métodos Se obtuvo información de fuente primaria y secundaria en los niveles de individuo, comunidad y región, sobre variables culturales, políticas y urbanas; esta información fue procesada en filtros orientados al análisis de compatibilidad y complementariedad entre niveles y variables.

Resultados La práctica de actividad física presenta aspectos relacionados que afectan el comportamiento individual, los significados de cuerpo, salud y autocuidado; los aspectos de ambiente urbano como el uso de suelo y la disposición de vías para desplazarse en bicicleta; y los desarrollos normativos como la promoción de políticas saludables orientadas a su fomento.

Conclusiones Se logró una aproximación cualitativa para el tratamiento de la información, sobre la práctica de actividad física en contextos urbanos, así como su interpretación con respecto a la construcción de relaciones sociales. Se identificaron aspectos comunes a las tres regiones y específicos para cada región. El enfoque multinivel permitió interpretar relaciones entre variables de nivel individual con otras de nivel político.

Palabras Clave: Actividad motora, multinivel, Colombia (fuente: DeCS, BIREME).

ABSTRACT

A multilevel approach to diagnosing physical activity in three parts of Colombia 
Objective Individual and contextual characteristics related to physical activity were studied to describe and interpret such practice in three parts of Colombian.

Methods Primary- and secondary-source information was obtained at individual, community and regional level concerning cultural, political and urban variables. This information was processed using filters orientated towards analysing compatibility and complementary aspects between levels and variables.

Results Practising physical activity presented related aspects affecting individual behaviour (the meaning of the body, health and looking after yourself), urban environmental aspects (soil use and the availability of bicycle-routes) and regulatory developments (health policy really orientated towards promoting health).

Conclusions A qualitative approach towards dealing with information about engaging in physical activity within an urban context, as well as interpreting such data respecting constructing social relationships, was achieved in this work. Common aspects for the three regions were identified, as were aspects specific for each region. The multilevel approach led to interpreting relationships between individual variables and political ones.

Key Words: physical activity, multilevel, Colombia (source: MeSH, NLM).

$\mathrm{E}$ n el fomento de la actividad física existen estudios que se han enfocado en el modelo ecológico (1), abordando los aspectos sociales, culturales y políticos que afectan su práctica. Se dirigen a identificar y comprender las relaciones que presentan los modos de vida con las maneras de enfermar y estar sano, identificando diferentes niveles de intervención; por ejemplo, algunos estudios consideran como niveles de influencia en los comportamientos saludables los factores personales, interpersonales y grupales, institucionales, comunitarios, y de política pública (2) y otros los niveles individual, organizacional y gubernamental, en los sectores escolar, laboral, hospitalario y comunitario (3). Aunque a estas teorías y modelos, se les ha atribuido la posibilidad de fundamentar en las personas y grupos, los mecanismos para la adopción y mantenimiento a largo plazo de hábitos saludables (2), sus métodos para generar las explicaciones de las relaciones sociales y culturales de la salud y de la enfermedad, aún se encuentran en construcción.

El enfoque multinivel ha sido una metodología útil para el desarrollo de diagnósticos y caracterizaciones de la actividad física desde una perspectiva ecológica. Se fundamenta en reconocer que existen procesos o condiciones de tipo social o político que pueden afectar las situaciones o comportamientos individuales; aproximándose a una interpretación de determinantes en 
salud, como factores esenciales, especialmente relacionados con los sistemas económico, político y jurídico (4), los cuales fijan límites o ejercen presión en los procesos de salud de las personas.

El origen del análisis multinivel se encuentra en las ciencias sociales especialmente en el campo de la educación, demografía y sociología (5-6) y se desarrolla en la salud pública a partir de la necesidad de establecer modelos causales de enfermedad, más rigurosos. En general su enfoque conceptual, ha sido usado para referirse al tratamiento jerárquico de la información (5) asociada con niveles cualitativos relevantes para entender la salud y la enfermedad. Dentro de sus posibilidades se encuentran los desarrollos estadísticos orientados al tratamiento cuantitativo de datos de cada grupo de variables de un nivel individual y otras de un nivel colectivo, conocido como método estadístico para el análisis multinivel (7).

El presente artículo expone los hallazgos obtenidos al aplicar un análisis con enfoque multinivel, como parte del desarrollo metodológico empleado en la caracterización de la actividad física en tres regiones de Colombia: Bogotá, Antioquia y Quindío. En ésta se definieron procesos de indagación de variables relacionadas al tema, establecidas por niveles de orden individual, comunitario-local y contextual.

Dicha caracterización fue parte del estudio "Modelos de movilización social con énfasis en la actividad física y estilos de vida saludables para reducir el sedentarismo en las regiones de Bogotá, D.C., Antioquia y Quindío”. El cual se ajustó a un enfoque ecológico social y tuvo como objetivo general diseñar, aplicar, evaluar y ajustar modelos de movilización social en salud que contribuyeran a incrementar los diversos tipos de actividad física y a reducir el sedentarismo, para prevenir enfermedades crónicas en hombres y mujeres entre 25 y 50 años en las tres regiones.

A continuación se exponen los procesos metodológicos empleados en la fase de análisis multinivel, los niveles de análisis y los hallazgos del mismo en la caracterización de la actividad física en las tres regiones enunciadas.

\section{MÉTODOS}

\section{Población de estudio}

Se definió una población objetivo para cada nivel y se indagaron variables específicas, conjugando técnicas cuantitativas y cualitativas para observarlas en poblaciones específicas de cada nivel abordado (Cuadros 1 y 2). 
60 REVISTA DE SALUD PUBLICA • Volumen 8 (Sup. 2), Noviembre 2006

\begin{tabular}{|c|c|c|c|}
\hline \multirow[b]{2}{*}{ Niveles } & \multicolumn{3}{|c|}{ Variables } \\
\hline & Política & Cultural & Equipamiento urbano \\
\hline Contextual & $\begin{array}{l}\text { Decisiones planes } \\
\text { de acción, con } \\
\text { orientación a la ac- } \\
\text { tividad física }\end{array}$ & $\begin{array}{l}\text { Procesos culturales propios de } \\
\text { Bogotá, Antioquia y Quindío }\end{array}$ & $\begin{array}{l}\text { Uso del suelo y } \\
\text { estructura urbana }\end{array}$ \\
\hline Técnica & $\begin{array}{l}\text { Entrevistas, } \\
\text { revisión } \\
\text { documental }\end{array}$ & Revisión documental & $\begin{array}{l}\text { Aforos, revisión } \\
\text { documental }\end{array}$ \\
\hline $\begin{array}{l}\text { Comunidad- } \\
\text { local }\end{array}$ & $\begin{array}{l}\text { Grados de } \\
\text { participación y } \\
\text { organización local, } \\
\text { redes, activos } \\
\text { comunitarios. } \\
\text { Procesos, } \\
\text { mecanismos y } \\
\text { necesidades } \\
\text { reconocidas por la } \\
\text { comunidad }\end{array}$ & $\begin{array}{l}\text { Representaciones sociales sobre } \\
\text { la actividad física, prácticas } \\
\text { comunitarias, tradiciones y } \\
\text { creencias y de riesgo de } \\
\text { enfermedad }\end{array}$ & $\begin{array}{l}\text { Representación } \\
\text { social de la } \\
\text { percepción de } \\
\text { presencia o ausencia } \\
\text { en el uso de recursos }\end{array}$ \\
\hline Técnica & $\begin{array}{l}\text { Entrevistas, } \\
\text { Inventario de } \\
\text { activos } \\
\text { comunitarios }\end{array}$ & $\begin{array}{l}\text { Entrevistas, observación en } \\
\text { terreno }\end{array}$ & Entrevistas \\
\hline Individuo & & $\begin{array}{l}\text { Prevalencia y actitudes ante la } \\
\text { práctica de actividad física }\end{array}$ & $\begin{array}{l}\text { Percepción de } \\
\text { ambiente y Hábitos } \\
\text { de movilidad }\end{array}$ \\
\hline Técnica & & Encuesta & Encuesta \\
\hline
\end{tabular}

Nivel Individual

Se aplicó una encuesta a hombres y mujeres entre 25 y 50 años de las tres regiones colombianas, en las que se estudiaron aspectos sociodemográficos, estado de salud y afiliación, nivel de actividad física en tiempo libre, ocupación y transporte, estadios de comportamiento frente a la actividad física, conocimientos sobre factores de riesgo y enfermedades crónicas, barreras ambientales para la actividad física. El muestreo se efectuó en cuatro etapas, estimando la muestra para cada región con una precisión del 2-3 \%, intervalos de confianza del 95 y $99 \%$. La muestra fue organizada en dos grupos: estudio y control.

\section{Nivel Contextual}

Se estudiaron aspectos relacionados con la dimensión política y de equipamiento urbano. En la primera se abordaron como categorías de análisis para cada región, las políticas orientadas al fomento de actividad física y los actores y escenarios políticos en que éstas operan; empleando como técnicas de investigación entrevistas, talleres y revisión de documentos. 
Análisis de datos

Articulación de la información. Una vez reunida la información de cada variable en las regiones, se efectuó la articulación de los hallazgos (8), para lo cual, se aplicaron criterios de compatibilidad (se entendió como la convergencia en hallazgos específicos sobre una variable a partir de los diferentes datos obtenidos entre niveles y dimensiones) y complementariedad (definida como la posibilidad de aumentar la información relacionada con una variable a partir de los diferentes datos existentes entre niveles y dimensiones). Se desarrollaron matrices de articulación de la información para cada región y posteriormente se relacionaron las tres regiones del estudio.

Cuadro 2. Población Objeto

\begin{tabular}{|c|c|c|c|}
\hline \multirow[b]{2}{*}{ Niveles } & \multicolumn{3}{|c|}{ Variables } \\
\hline & Política & Cultural & Equipamiento urbano \\
\hline Contextual & $\begin{array}{l}55 \text { instituciones de diferentes } \\
\text { sectores: salud, educación, } \\
\text { recreación y deporte, entre otros } \\
\text { (Bogotá: } 21 \text {, Antioquia: 19, } \\
\text { Quindío: } 15 \text { ) }\end{array}$ & Revisión documental & Revisión documental \\
\hline $\begin{array}{l}\text { Comunidad- } \\
\text { local }\end{array}$ & $\begin{array}{l}\text { Inventario de activos en } \\
\text { comunidades ( } 6 \text { Bogotá, } 4 \\
\text { Antioquia y } 5 \text { en Quindío) }\end{array}$ & $\begin{array}{l}24 \text { barrios } 8 \text { por } \\
\text { región } \\
130 \text { personas } \\
\text { entrevistadas }\end{array}$ & $\begin{array}{l}\text { Aforos en barrios: } \\
8 \text { en Bogotá, } 4 \text { en } \\
\text { Antioquia y } 4 \text { en } \\
\text { Quindío }\end{array}$ \\
\hline Individuo & & $\begin{array}{l}3998 \text { personas encue } \\
\text { Antioquia } 1602 \text { y Quin }\end{array}$ & $\begin{array}{l}\text { tadas: Bogotá 1600, } \\
\text { dío } 1196 .\end{array}$ \\
\hline
\end{tabular}

Panorama de la problemática. Consistió en un análisis cualitativo, efectuado a partir de los hallazgos de compatibilidad y complementariedad, orientado a la interpretación de relaciones entre regiones y los aspectos que se identificaron comunes y diferentes en las mismas.

Una vez se elaboró el panorama se analizaron los aspectos críticos y fueron sometidos a un ejercicio de interpretación conceptual que condujo posteriormente a la organización o clasificación de los hallazgos que afectaban la práctica de actividad física.

\section{RESULTADOS}

A continuación se presenta una síntesis de los aspectos relevantes encontrados en los niveles individual, local y contextual.

Nivel individual

De manera general, se encontró que el 50,1 \% de la población encuestada eran mujeres, el 63,4 \% tenían como nivel educativo la formación secundaria 
y el $24 \%$ nivel universitario; el $65 \%$ reportaron como ingreso hasta dos salarios mínimos, siendo el 38,9 \% trabajadores independientes, el 31,5 \% empleados y el 13,5 \% desempleados.

Con relación al nivel de actividad física, se encontró que el 65,7 \% en el grupo control y el 59,3 \% en el grupo de estudio refieren un estado de insuficientemente activo, durante los tiempos libre y en el hogar. Es decir, que las personas encuestadas no practican actividad física al menos cinco días a la semana, y consumen menos de 600 METs totales ${ }^{1}$, en este periodo.

Nivel local

En cada región se encontraron aspectos específicos. En Bogotá, se identificó que los grupos interpretan el cuerpo desde una noción instrumental asociada con el uso de éste para fines de producción e ingreso. En Antioquia, se encontró una noción de cuerpo centrada en el lenguaje, como dispositivo social y la percepción de inseguridad y violencia como limitación para el uso del espacio público; los grupos refirieron un sentido de arraigo territorial y de construcción de hábitos (sentido de pertenencia). En Quindío también se identificó un significado del cuerpo desde una noción instrumental y un sentido de pertenencia territorial.

En las tres regiones se identificó que en aquellas comunidades que contaban con menor presencia de autoridades locales o que estaban menos organizadas, las personas entrevistadas percibían sus ambientes con mayor inseguridad y evidencia de pobreza (estado de viviendas y espacio público) e interpretaban la práctica de actividad física como una posibilidad de afianzar la interacción social.

Especialmente en Bogotá y con menor fuerza en Quindío, los grupos estudiados manifestaron desconfianza en sus líderes y no refirieron conocer las acciones de autoridades locales, en temas relacionados con el fomento de la actividad física.

Nivel contextual

Como principales hallazgos en esta dimensión se encontraron para Bogotá, la ausencia de una política integrada de actividad física; el predominio de un enfoque de riesgo y preventivo; un interés creciente por el tema de la activi-

1 METs: Equivalente metábolico. Un METs equivale a 1,2 kcal/min para un individuo de 70 kilos de peso, correspondiente al estado de reposo 
dad física, el reconocimiento de las disposiciones normativas que soportan las acciones institucionales, la fragmentación de acciones sectoriales, intereses diversos y no coincidentes y la duplicidad en los esfuerzos y acciones. En cuanto a los escenarios políticos algunos eran propiciados por las autoridades del distrito, también se observaron interacciones en ambientes de nivel nacional relacionados con el sector del deporte o el de salud.

En Antioquia se identificó la articulación de dos sectores, deporte y salud, en relación con la organización de un conjunto de acciones para el fomento de la actividad física; no obstante en la ciudad capital, Medellín, se encontró un conjunto de acciones menos articuladas, provenientes de diferentes sectores. En los actores políticos se observó sensibilidad al tema y compromiso en su desarrollo al adelantar esfuerzos en relación con la articulación de acciones. Con relación a los escenarios existen algunos espacios comunes entre actores para la participación en el tema e iniciativas la construcción de los mismos con mayor rigor y sostenibilidad en el largo plazo.

Para la región de Quindío, se identificó que un interés para el desarrollo de acciones y políticas en relación con el fomento de la actividad física. Los actores interesados en el fomento de la actividad física provienen de diferentes sectores tanto públicos, como privados. Aunque no se encontraron escenarios comunes para el desarrollo político, en el proceso de diagnóstico, los actores manifestaron su interés en consolidar un trabajo articulado e intersectorial.

Con relación al equipamiento urbano en general, se identificó que Quindío no cuenta con condiciones urbanas favorables, para la actividad física, Antioquia, solamente en un sector evaluado y Bogotá en cuatro de los ocho evaluados.

Panorama de la práctica de actividad física

Se identificaron los aspectos comunes a las tres regiones encontrando en primer lugar, un énfasis por parte de las instituciones que fomentan la actividad física, en los sectores de salud o de recreación y deporte, en la capacidad de transformar las actitudes y prácticas de las personas, desde una orientación centrada en los niveles de orden individual e interpersonal y basadas en la capacidad de decisión que éstas tienen.

La anterior situación resultó en parte, antagónica con la presencia de los problemas socioeconómicos, y de equipamiento urbano asociados en las tres regiones; así como, la pérdida de autonomía en la capacidad de transforma- 
ción de la realidad (autoeficacia individual y colectiva) en la medida en que se tiene mayor nivel de escolaridad.

A partir del anterior análisis por región, se presenta en el Cuadro 3, los elementos que para el estudio se interpretaron como aspectos representativos que caracterizan la práctica de actividad física en las regiones.

Cuadro 3. Elementos representativos de las regiones

\begin{tabular}{|c|c|c|c|}
\hline ELEMENTOS & BOGOTÁ & ANTIOQUIA & QUINDÍO \\
\hline $\begin{array}{l}\text { Contextos desfavorables con las condiciones } \\
\text { de baja presencia institucional, pobreza e } \\
\text { inseguridad }\end{array}$ & SI & SI & SI \\
\hline $\begin{array}{l}\text { La práctica de actividad física, percibida } \\
\text { como la posibilidad de afianzar la interacción } \\
\text { social }\end{array}$ & SI & $\mathrm{SI}$ & SI \\
\hline $\begin{array}{l}\text { Respuesta institucional, basada en enfoques } \\
\text { de orientación individual e interpersonal, y en } \\
\text { estrategias orientadas a afianzar los } \\
\text { conocimientos sobre el autocuidado y la } \\
\text { actividad física. }\end{array}$ & SI & $\begin{array}{l}\mathrm{SI} \\
\mathrm{NO} \text { (en } \\
\text { algunos } \\
\text { casos) }\end{array}$ & SI \\
\hline $\begin{array}{l}\text { Interpretación del cuerpo desde una noción } \\
\text { instrumental }\end{array}$ & SI & NO & SI \\
\hline $\begin{array}{l}\text { Noción de cuerpo centrada en el lenguaje, } \\
\text { como dispositivo social }\end{array}$ & NO & SI & NO \\
\hline Arraigo territorial y habitar & NO & SI & SI \\
\hline $\begin{array}{l}\text { Actuación hegemónica de actores políticos, } \\
\text { baja participación social y el desarraigo }\end{array}$ & SI & NO & NO \\
\hline Violencia y uso del espacio público & NO & SI & NO \\
\hline
\end{tabular}

A manera de ejercicio estos elementos comunes se exponen en una red de relaciones (Figura 1) que intenta esquematizar cómo la práctica de actividad física se expresa a través de los niveles y dimensiones.

Esta primera interpretación permitió identificar tres tipos de núcleos críticos: los de carácter estructural, los de carácter primario y los de carácter organizativo. Los estructurales hacen relación a las condiciones que dificultan el desarrollo de actividad física, en el nivel contextual, es decir por aspectos sociales, económicos, culturales, políticos y ambientales manifestados en las regiones. Los primarios, se relacionan con los atributos negativos para la práctica de actividad física, presentes en las personas y comunidades, producto del contexto y de su propia capacidad de elección. Los organizativos, tienen que ver con las maneras como se responde al problema desde institu- 
ciones e iniciativas particulares. En este sentido, se observó que las acciones generadas por parte de las instituciones y autoridades responsables del tema, se dirigen especialmente a la atención de los núcleos primarios.

Figura 1. Relaciones existentes que explican la problemática de la práctica de actividad física, de acuerdo con los avances en la fase de investigación.

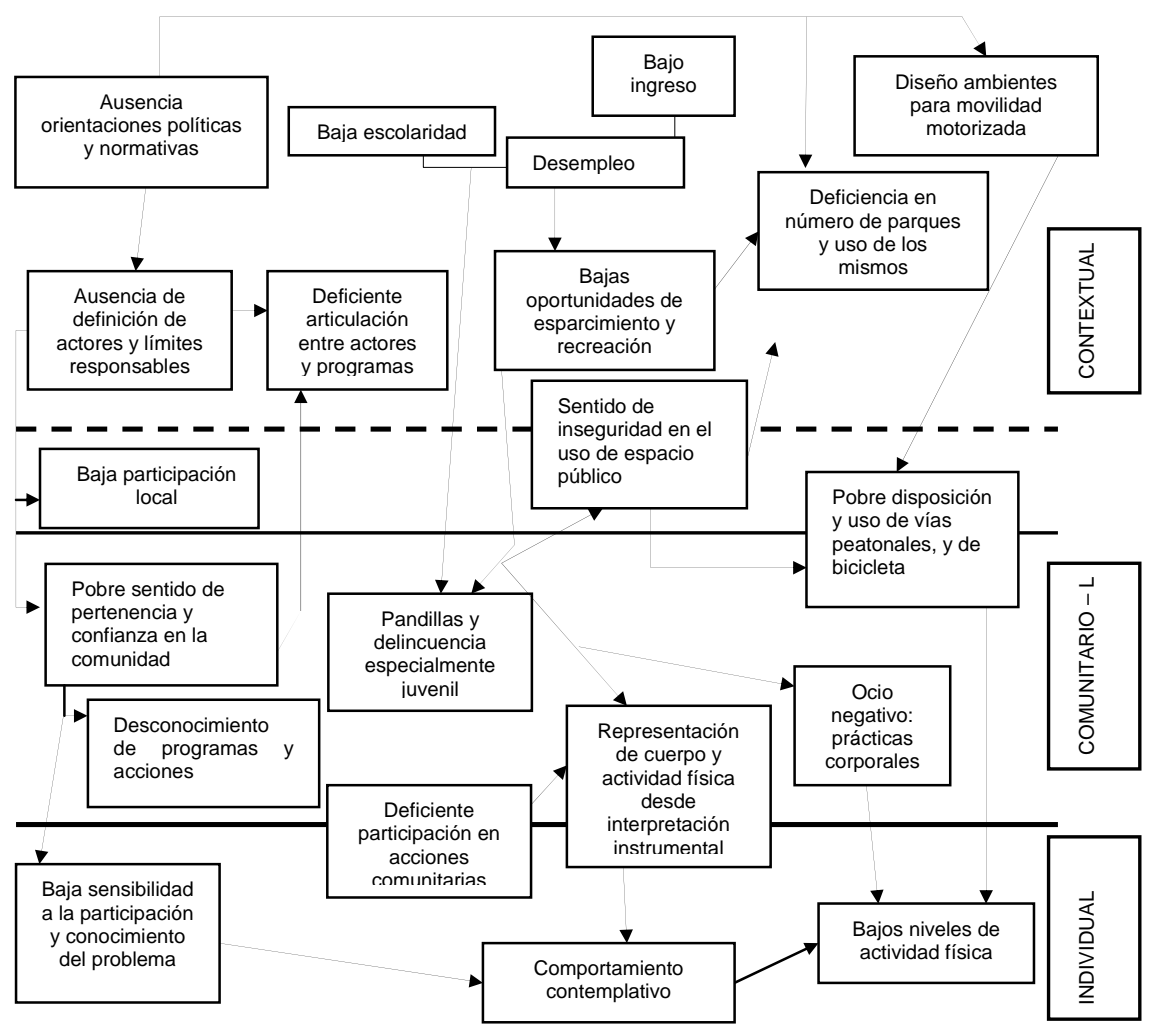

\section{Núcleos estructurales}

1. Deficiente estructuración de procesos de integración de la definición del problema de actividad física, a una política pública. Sin un escenario que le de lugar a la acción articulada intersectorialmente y sin establecimiento claro de responsabilidades, recursos e indicadores.

2. Deficiente conformación de espacios o entornos que induzcan a la práctica de actividad física en los tiempos de movilización o transporte y en los de esparcimiento y recreación. 
3. Bajas oportunidades de esparcimiento y recreación producto de condiciones de pobreza, escolaridad baja y bajo ingreso.

Núcleos organizativos

1. Duplicidad en el desarrollo de esfuerzos y uso de recursos, por parte de las acciones, debido a deficiente comunicación y comprensión articulada del problema.

2. Oferta de programas centrados en la acción en el ámbito individual y atendiendo a estrategias de información y desarrollo de eventos.

3. Ausencia de sistema de información para la vigilancia del sedentarismo y el seguimiento de la actividad física.

4. Ausencia de estructura de costos para la implementación y sostenimiento de acciones orientadas a la actividad física.

5. Métodos de evaluación del modelo de promoción.

Núcleos primarios

1. Deficientes procesos de participación y organización comunitaria

2. Prácticas corporales no saludables

3. Desconocimiento de la inactividad física como problema, para la vida

\section{DISCUSIÓN}

La asociación de la práctica de actividad física con las relaciones sociales identificada en el estudio, también se encuentra en otros trabajos como el desarrollado por Baker y cols (9), en el cual se plantea que las personas que disponen de una red de amigos y familiares más amplia son más activas que aquellas que no cuentan con esa condición. Igualmente, identificó categorías como la capacidad comunitaria y el sentido de pertenencia a una comunidad con aspectos favorables para la práctica de actividad física; en este sentido, la presente investigación encontró resultados similares para las regiones en las que las personas referían una mayor confianza en sus líderes y comunidades.

Por otra parte, existen interrogantes relacionados con la estructura organizativa y el desarrollo comunitario en relación con la práctica de actividad física; especialmente la presencia de comunidades más activas cuando cuentan con soporte social y de autoridades locales y si es la capacidad comunitaria, la que conduce a una mayor presencia social y de autoridades locales. En este sentido según Starfield, el fortalecimiento de la atención pri- 
maria y de las redes de apoyo social conduciría a un efecto a nivel individual relacionado con una mayor confianza y percepción de salud (10).

Existen de igual forma estudios que asocian las condiciones de diseño urbano con la práctica de actividad física entre ellos el de Simmons-Morton y cols. En el que se destaca la promoción de actividad física a través del establecimiento de ciclorutas, parques e incentivos para desplazarse al trabajo caminando o en bicicleta (11). Aspecto que también fue identificado en el presente estudio, especialmente en relación con la baja favorabilidad para la práctica de actividad física en Antioquia y Quindío.

Finalmente, la caracterización de la práctica de actividad física desde un enfoque multinivel, permite identificar una diversidad de aspectos que influyen y se presentan simultáneamente en la realidad de las personas y de las comunidades, lo cual deriva a la necesidad de establecer planes de acción articulados e intersectoriales.

El uso de análisis multinivel es una estrategia de investigación útil en el tratamiento de información, aún cuando no se logró incorporar al mismo, aspectos estadísticos y cuantitativos para establecer asociaciones entre niveles. En la presente investigación, este enfoque favoreció la comprensión de la práctica de actividad física en un sentido amplio y condujo a la planeación de acciones para su fomento en diferentes dimensiones y niveles.

Se considera por tanto que el enfoque multinivel, es de ayuda para la investigación de condiciones de riesgo y protectoras de la salud, en las cuales el comportamiento humano está de por medio y la organización y estructura del contexto en que este se encuentra son aspectos influyentes.

En conclusión, este trabajo aporta en el estudio de la actividad física desde un enfoque más amplio que el individual e ilustra en el contexto colombiano, algunos aspectos que al parecer se encuentran relacionados y por tanto deben ser considerados al fomentar esta práctica •

Agradecimientos. Los autores expresan su agradecimiento a las instituciones financiadoras del proyecto "Modelos de movilización social con énfasis en la actividad física y estilos de vida saludables para reducir el sedentarismo en las regiones de Bogotá, Antioquia y Quindío”: Ministerio de Protección Social, Instituto Colombiano para el Desarrollo de la Ciencia y la Tecnología "Francisco José de CaldasColciencias", Universidad Nacional de Colombia y a la Fundación Ciudad Humana. Código: 1101-04-14483. 
68 REVISTA DE SALUD PUBLICA • Volumen 8 (Sup. 2), Noviembre 2006

\section{REFERENCIAS}

1. U.S.Department of Health and Human Services. Physical Activity and Health: A Report of the Surgeon General. Atlanta: USDHSS/CDC; 1996.

2. McLeroy KR, Bibeau D, Steckler A, Glanz K. An ecological perspective on health promotion programs. Health Education Quarterly 1988; 15: 351377.

3. Simons-Morton DB, Simons-Morton BG, Parcel GS, Bunker JF. Influencing personal and environmental conditions for community health: a multilevel intervention model. Community Health 1988; 11: 25-35.

4. Krieger N. Glosario de Epidemiología Social (parte II). En: Boletín Epidemiológico / OPS 2002; 23 (2):10-13.

5. Diez-Roux A. Multilevel analysis in public health research. En: Annu. Rev. Public Health. 2000; 21:171-192.

6. Diez-Roux A. Glosario de análisis multinivel, parte I. En: Boletín epidemiológico / OPS, 2003; 24(3):11-13.

7.Diez-Roux A. Glosario de análisis multinivel, parte II. En: Boletín epidemiológico / OPS, 2003; 24(4):10-13.

8. Prieto A. A model of health promotion with emphasis on physical activity for university students. Rev. Salud Pública, Sept./Dec. 2003; 5(3): 284-300.

9. Baker E, Brennan L, Houseman R. Measuring the determinants of physical activity in the community: current and future directions. Research Quaterly for Exercise and Sport, Washington 2000; 71(2):146-59.

10. Starfield B. Equidad en salud y atención primaria: una meta para todos. En: Gerencia y políticas de salud 2000; 11.

11. Simons-Morton DB, Simons-Morton BG, Parcel GS, Bunker JF. Influencing personal and environmental conditions for community health: a multilevel intervention model. Community Health 1988; 11: 25-35. 